PROCEEDINGS OF THE

AMERICAN MATHEMATICAL SOCIETY

Volume 136, Number 6, June 2008, Pages 2193-2200

S 0002-9939(08)09261-7

Article electronically published on January 17, 2008

\title{
POINTWISE HARDY INEQUALITIES AND UNIFORMLY FAT SETS
}

\author{
JUHA LEHRBÄCK
}

(Communicated by Juha M. Heinonen)

\begin{abstract}
We prove that it is equivalent for domain in $\mathbb{R}^{n}$ to admit the pointwise $p$-Hardy inequality, have uniformly $p$-fat complement, or satisfy a uniform inner boundary density condition.
\end{abstract}

\section{INTRODUCTION}

We say that a domain $\Omega \subset \mathbb{R}^{n}$ admits the pointwise $p$-Hardy inequality for $1<p<\infty$ if there exists $1<q<p$ such that the inequality

$$
|u(x)| \leq C d_{\Omega}(x)\left(\sup _{r \leq 2 d_{\Omega}(x)} \frac{1}{|B(x, r)|} \int_{B(x, r)}|\nabla u(y)|^{q} d y\right)^{1 / q}
$$

holds for all $u \in C_{0}^{\infty}(\Omega)$ and all $x \in \Omega$ with a constant $C=C(\Omega, n, p, q)>0$; here $d_{\Omega}(x)$ denotes the distance from $x \in \Omega$ to the boundary of $\Omega$. These inequalities were introduced by Hajłasz in [2, but Kinnunen and Martio also considered similar inequalities independently in [6. It was proved in 2] (see also [6]) that if $1<p<\infty$ and the complement of the domain $\Omega \subset \mathbb{R}^{n}$ is sufficiently big, namely uniformly $p$-fat (see Section 2 for precise definitions), then $\Omega$ admits the pointwise $p$-Hardy inequality. Notice that it follows immediately from the definition that if $1<p_{0}<\infty$ and a domain $\Omega$ admits the pointwise $p_{0}$-Hardy inequality, there exists $1<q<p_{0}$ such that $\Omega$ admits pointwise $p$-Hardy inequalities for all $p>q$.

If $u \in C_{0}^{\infty}(\Omega)$ is such that (1) holds for all $x \in \Omega$ with a constant $C_{1}>0$, it is easy to see, using the Hardy-Littlewood-Wiener maximal function theorem, that $u$ satisfies the usual $p$-Hardy inequality

$$
\int_{\Omega}|u(x)|^{p} d_{\Omega}(x)^{-p} d x \leq C \int_{\Omega}|\nabla u(x)|^{p} d x
$$

with a constant $C=C\left(C_{1}, n, p\right)>0$. This classical inequality was first considered in the one-dimensional case by Hardy (cf. 3] and references therein). Nečas 9 generalized $p$-Hardy inequalities to higher dimensions when he proved that, for all $1<p<\infty$, the inequality (2) holds in a bounded Lipschitz domain $\Omega \subset \mathbb{R}^{n}$ for all $u \in C_{0}^{\infty}(\Omega)$, with a constant $C=C(\Omega, n, p)>0$ (i.e. $\Omega$ admits the $p$-Hardy inequality). Later Ancona (the case $p=2$ ) 1, Lewis 8, and Wannebo 11 proved that a domain $\Omega \subset \mathbb{R}^{n}$ admits the $p$-Hardy inequality under the assumption that the

Received by the editors May 16, 2007.

2000 Mathematics Subject Classification. Primary 46E35, 31C15; Secondary 26D15, 42B25.

The author was supported in part by the Academy of Finland.

(c)2008 American Mathematical Society Reverts to public domain 28 years from publication 
complement of $\Omega$ is uniformly $p$-fat. Recall that in [2] and [6] this same assumption was shown to be sufficient for $\Omega$ to admit even the pointwise $p$-Hardy inequality. We also remark that the complement of a proper subdomain $\Omega \subsetneq \mathbb{R}^{n}$ is uniformly $p$-fat for all $p>n$.

However, the pointwise $p$-Hardy inequality is not equivalent to the usual $p$-Hardy inequality, since there are domains which admit the latter for some $p$, but where the corresponding pointwise inequality fails to hold. In particular, it is not true that the $p_{0}$-Hardy inequality would imply $p$-Hardy inequalities for all $p>p_{0}$, as is the case with pointwise inequalities. This can be seen by considering e.g. the punctured unit ball $B(0,1) \backslash\{0\} \subset \mathbb{R}^{n}$, which admits the pointwise $p$-Hardy inequality only in the trivial case $p>n$, but where the usual $p$-Hardy inequality also holds when $1<p<n$; yet the $n$-Hardy inequality fails in this domain. This example also shows that the uniform $p$-fatness of the complement is not necessary for a domain to admit the $p$-Hardy inequality, as the complement of $B(0,1) \backslash\{0\} \subset \mathbb{R}^{n}$ is not uniformly $p$-fat for any $p \leq n$. Nevertheless, as a part of our main theorem, we show that uniform $p$-fatness of $\Omega^{c}$ is not only sufficient, but also necessary for $\Omega$ to admit the pointwise $p$-Hardy inequality.

We say that a domain $\Omega \subset \mathbb{R}^{n}$ satisfies an inner boundary density condition with exponent $\lambda$, if there exists a constant $C>0$ such that

$$
\mathcal{H}_{\infty}^{\lambda}\left(B\left(x, 2 d_{\Omega}(x)\right) \cap \partial \Omega\right) \geq C d_{\Omega}(x)^{\lambda} \text { for every } x \in \Omega .
$$

It turns out that condition (3), for some exponent $\lambda>n-p$, is also necessary and sufficient for a domain $\Omega \subset \mathbb{R}^{n}$ to admit the pointwise $p$-Hardy inequality, and hence equivalent to the uniform $p$-fatness of $\Omega^{c}$. Let us now formulate our main result.

Theorem 1. Let $\Omega \subset \mathbb{R}^{n}$ be a domain and let $1<p<\infty$. Then the following conditions are equivalent:

(a) The complement $\Omega^{c}$ is uniformly p-fat.

(b) $\Omega$ admits the pointwise $p$-Hardy inequality.

(c) There exists $n-p<\lambda \leq n$ such that $\Omega$ satisfies the inner boundary density condition (3) with the exponent $\lambda$.

Theorem 1 can be considered as an extension of the result, proved by Ancona [1] $(n=2)$ and Lewis [8], that a domain $\Omega \subset \mathbb{R}^{n}$ admits the $n$-Hardy inequality if and only if the complement of $\Omega$ is uniformly $n$-fat.

Results related to Theorem 1 were also considered in [7, where the following local dichotomy was shown: Suppose that a domain $\Omega \subset \mathbb{R}^{n}$ admits the $p$-Hardy inequality and let $w \in \partial \Omega, r>0$. Then either the Hausdorff dimension of $B(w, r) \cap$ $\partial \Omega$ is strictly larger that $n-p$, or the Minkowski dimension of $B(w, r) \cap \partial \Omega$ is strictly less than $n-p$. Now, if $\Omega$ admits the pointwise $p$-Hardy inequality, we obtain, by Theorem 11. that only the former of the two possibilities above may occur. Indeed, when $w \in \partial \Omega$ and $r>0$, there exists $x \in B(w, r / 3) \cap \Omega$, whence $B\left(x, 2 d_{\Omega}(x)\right) \subset B(w, r)$, and thus

$$
\operatorname{dim}_{\mathcal{H}}(B(w, r) \cap \partial \Omega) \geq \operatorname{dim}_{\mathcal{H}}\left(B\left(x, 2 d_{\Omega}(x)\right) \cap \partial \Omega\right) \geq \lambda>n-p .
$$

\section{Preliminaries}

When $A$ is a subset of the $n$-dimensional Euclidean space $\mathbb{R}^{n}, \partial A$ denotes the boundary of $A$ and $A^{c}=\mathbb{R}^{n} \backslash A$ is the complement of $A$. The characteristic 
function of $A$ is $\chi_{A}$, and $|A|$ denotes the $n$-dimensional Lebesgue measure of $A$. The Euclidean distance between two points, or a point and a set, is denoted $d(\cdot, \cdot)$. When $\Omega$ is a domain, i.e. an open and connected set, and $x \in \Omega$, we also use notation $d_{\Omega}(x)=d(x, \partial \Omega)$. An open ball with center $x \in \mathbb{R}^{n}$ and radius $r>0$ is denoted $B(x, r)$, and the corresponding closed ball is $\bar{B}(x, r)$. If $B=B(x, r)$ and $L>0$, we denote $L B=B(x, L r)$. The support of a function $u: \Omega \rightarrow \mathbb{R}$, spt $(u)$, is the closure of the set where $u$ is non-zero. We let $C$ denote various positive constants, which may vary from expression to expression.

The restricted Hardy-Littlewood maximal function of $f \in L_{\text {loc }}^{1}\left(\mathbb{R}^{n}\right)$ is defined by

$$
M_{R} f(x)=\sup _{0<r \leq R} \frac{1}{|B(x, r)|} \int_{B(x, r)}|f(y)| d y .
$$

The well-known maximal function theorem of Hardy, Littlewood and Wiener (see e.g. [10]) states that if $1<p<\infty$, we have $\left\|M_{R} f\right\|_{p} \leq C(n, p)\|f\|_{p}$ for all $0<$ $R \leq \infty$. When $1<q<\infty$, we denote $M_{R, q} f=\left(M_{R} f^{q}\right)^{1 / q}$. Using this notation, we may now write the pointwise $p$-Hardy inequality (11) as

$$
|u(x)| \leq C d_{\Omega}(x) M_{2 d_{\Omega}(x), q}(|\nabla u|)(x),
$$

where $1<q<p$.

The $\lambda$-Hausdorff content of a set $A \subset \mathbb{R}^{n}$ is

$$
\mathcal{H}_{\infty}^{\lambda}(A)=\inf \left\{\sum_{i=1}^{\infty} r_{i}^{\lambda}: A \subset \bigcup_{i=1}^{\infty} B\left(z_{i}, r_{i}\right)\right\},
$$

where $z_{i} \in A$ and $r_{i}>0$. The Hausdorff dimension of $A \subset \mathbb{R}^{n}$ is then

$$
\operatorname{dim}_{\mathcal{H}}(A)=\inf \left\{\lambda>0: \mathcal{H}_{\infty}^{\lambda}(A)=0\right\} .
$$

We say that the boundary of a domain $\Omega \subset \mathbb{R}^{n}$ is $\lambda$-thick, if there exists a constant $C>0$ such that

$$
\mathcal{H}_{\infty}^{\lambda}(B(w, r) \cap \partial \Omega) \geq C r^{\lambda}
$$

for all $w \in \partial \Omega$ and $0<r<\operatorname{diam}(\Omega)$. It is clear that $\lambda$-thickness of $\partial \Omega$ implies that condition (3) holds in $\Omega$. The converse however is not true; see Section 4 for an example.

Let $\Omega \subset \mathbb{R}^{n}$ be a domain. The $p$-capacity of a compact set $E \subset \Omega$ (relative to $\Omega)$ is defined as

$$
\operatorname{cap}_{p}(E, \Omega)=\inf \left\{\int_{\Omega}|\nabla u|^{p} d x: u \in C_{0}^{\infty}(\Omega), u \geq 1 \text { on } E\right\} .
$$

A closed set $E \subset \mathbb{R}^{n}$ is said to be uniformly $p$-fat if there exists a constant $C>0$ such that

$$
\operatorname{cap}_{p}(E \cap \bar{B}(x, r), B(x, 2 r)) \geq C \operatorname{cap}_{p}(\bar{B}(x, r), B(x, 2 r))
$$

for all $x \in E$ and $r>0$. Note that for each ball $B(x, r) \subset \mathbb{R}^{n}$ we have

$$
\operatorname{cap}_{p}(\bar{B}(x, r), B(x, 2 r))=C(n, p) r^{n-p} .
$$

For this and other basic properties of the $p$-capacity we refer to [4].

We record the following useful lemma between Hausdorff content and $p$-capacity; for a proof, see e.g. [5, Thm. 5.9]. 
Lemma 2. Let $E \subset B(x, r) \subset \mathbb{R}^{n}$ be a compact set such that

$$
\mathcal{H}_{\infty}^{\lambda}(E) \geq C_{1} r^{\lambda}
$$

for some $\lambda>n-p$ and $C_{1}>0$. Then

$$
\operatorname{cap}_{p}(E \cap B(x, r), \bar{B}(x, 2 r)) \geq C r^{n-p}
$$

where $C=C\left(C_{1}, n, p\right)>0$.

\section{Proof of Theorem 1}

The part $(a) \Longrightarrow(b)$ of Theorem 1 is contained in [2, Thm. 2]; the proof of this part relies on the self-improving property of $p$-fatness, due to Lewis [ 8 , Thm. 1]. Let us now prove the implications $(b) \Longrightarrow(c)$ and $(c) \Longrightarrow(a)$ to obtain the equivalence of the conditions in the theorem.

Proof of $(b) \Longrightarrow(c)$. Let $\Omega \subset \mathbb{R}^{n}$ and $1<p<\infty$. We assume that condition (3) fails for every $n-p<\lambda \leq n$, and show that then the pointwise $p$-Hardy inequality fails in $\Omega$ as well. To this end, let $1<q<p$ and choose $\lambda=n-q>n-p$. It is evident that (3) is equivalent to the condition that there exists some $C_{1}>0$ such that

$$
\mathcal{H}_{\infty}^{\lambda}\left(\bar{B}\left(x, 3 d_{\Omega}(x)\right) \cap \partial \Omega\right) \geq C_{1} d_{\Omega}(x)^{\lambda} \text { for every } x \in \Omega .
$$

Since (5) now fails for the chosen $\lambda$, there exist, for each $k \in \mathbb{N}$, a point $x_{k} \in \Omega$ such that

$$
\mathcal{H}_{\infty}^{\lambda}\left(E_{k}\right)<k^{-1} R_{k}^{\lambda}
$$

where we denote $R_{k}=d_{\Omega}\left(x_{k}\right)$ and $E_{k}=\bar{B}\left(x_{k}, 3 R_{k}\right) \cap \partial \Omega$. Using this, and the fact that $E_{k}$ is compact, we find, for a fixed $k \in \mathbb{N}$, a finite covering $\left\{B_{i}\right\}_{i=1}^{N}, B_{i}=$ $B\left(w_{i}, r_{i}\right)$ with $w_{i} \in \partial \Omega$ and $r_{i}>0$, such that $E_{k} \subset \bigcup_{i=1}^{N} B_{i}$ and $\sum_{i=1}^{N} r_{i}^{\lambda}<k^{-1} R_{k}^{\lambda}$.

Define a function $\varphi_{k}$ by

$$
\varphi_{k}(x)=\min _{1 \leq i \leq N}\left\{1, r_{i}^{-1} d\left(x, 2 B_{i}\right)\right\}
$$

and let $\psi_{k} \in C_{0}^{\infty}\left(B\left(x_{k}, 3 R_{k}\right)\right)$ be such that $0 \leq \psi_{k} \leq 1$ and $\psi_{k}(x)=1$ for all $x \in B\left(x_{k}, 2 R_{k}\right)$. Then $u_{k}=\psi_{k} \varphi_{k} \chi_{\Omega}$ is a Lipschitz function with compact support in $\Omega$. Since $r_{i}<k^{-1 / \lambda} R_{k}$ for all $1 \leq i \leq N$, we have that

$$
d\left(x_{k}, 3 B_{i}\right)>\frac{1}{4} R_{k}>r_{i}
$$

for all $1 \leq i \leq N$ if $k>4^{\lambda}$, and hence $u_{k}\left(x_{k}\right)=1$ for these $k$.

Next, denote $A_{i}=3 \bar{B}_{i} \backslash 2 B_{i}$. Then $\operatorname{spt}\left(\left|\nabla u_{k}\right|\right) \cap B\left(x_{k}, 2 R_{k}\right) \subset \bigcup_{i=1}^{N} A_{i}$ and we have in fact for a.e. $y \in B\left(x_{k}, 2 R_{k}\right)$ that

$$
\left|\nabla u_{k}(y)\right|^{q} \leq \sum_{i=1}^{N} r_{i}^{-q} \chi_{A_{i}}(y) .
$$

Let us now estimate the right-hand side of the pointwise $p$-Hardy inequality (4) at $x_{k}$. Since $\operatorname{spt}\left(\left|\nabla u_{k}\right|\right) \cap B\left(x_{k}, 2 R_{k}\right) \subset \bigcup_{i=1}^{N} 3 \bar{B}_{i}$, it follows from (6) that we must 
have $r>\frac{1}{4} R_{k}$ in order to obtain something positive when estimating the maximal function of $\left|\nabla u_{k}\right|$ at $x_{k}$. Hence, using (7), we calculate

$$
\begin{aligned}
& M_{2 R_{k}}\left(\left|\nabla u_{k}\right|^{q}\right)\left(x_{k}\right) \leq C \sup _{\frac{1}{4} R_{k} \leq r \leq 2 R_{k}}\left(r^{-n} \int_{B\left(x_{k}, r\right)}\left|\nabla u_{k}(y)\right|^{q} d y\right) \\
& \quad \leq C R_{k}^{-n} \int_{B\left(x_{k}, 2 R_{k}\right)}\left|\nabla u_{k}(y)\right|^{q} d y \leq C d_{\Omega}\left(x_{k}\right)^{-n} \sum_{i=1}^{N}\left|A_{i}\right| r_{i}^{-q} \\
& \quad \leq C d_{\Omega}\left(x_{k}\right)^{-n} \sum_{i=1}^{N} r_{i}^{n-q} .
\end{aligned}
$$

Recall that $\lambda=n-q>n-p$ and that $\sum_{i=1}^{N} r_{i}^{\lambda}<k^{-1} d_{\Omega}\left(x_{k}\right)^{\lambda}$. Thus

$$
\begin{gathered}
d_{\Omega}\left(x_{k}\right)^{q} M_{2 R_{k}}\left(\left|\nabla u_{k}\right|^{q}\right)\left(x_{k}\right) \leq C d_{\Omega}\left(x_{k}\right)^{q-n} \sum_{i=1}^{N} r_{i}^{n-q} \\
\leq C d_{\Omega}\left(x_{k}\right)^{-\lambda} k^{-1} d_{\Omega}\left(x_{k}\right)^{\lambda} \leq \frac{C}{k},
\end{gathered}
$$

and so the right-hand side of the inequality (4) for $u_{k}$ at $x_{k}$ tends to zero as $k \rightarrow \infty$. However, $u_{k}\left(x_{k}\right)=1$ for large $k$, so the pointwise $p$-Hardy inequality fails to hold with a uniform constant for all compactly supported Lipschitz functions in $\Omega$. By a standard approximation argument it is then clear that $\Omega$ does not admit the pointwise $p$-Hardy inequality.

Proof of $(c) \Longrightarrow(a)$. There now exists $n-p<\lambda \leq n$ so that $\Omega$ satisfies the density condition (3) with the exponent $\lambda$ and with a constant $C_{1}>0$. To prove that $\Omega^{c}$ is uniformly $p$-fat, it is in fact enough to show that there exists a constant $C=C\left(C_{1}, n, \lambda\right)>0$ such that

$$
\mathcal{H}_{\infty}^{\lambda}\left(B(w, r) \cap \Omega^{c}\right) \geq C r^{\lambda}
$$

for all $w \in \partial \Omega$ and $r>0$. Indeed, assume that (8) holds for all $w \in \partial \Omega$ and let $z \in \Omega^{c}, r>0$. If $B(z, r / 2) \subset \Omega^{c}$, then it easily follows (compare to calculations in (10) below) that (8) also holds for the ball $B(z, r)$, with a constant depending only on $n$. On the other hand, if $B(z, r / 2) \cap \Omega \neq \emptyset$, there is $w \in \partial \Omega$ such that $B(w, r / 2) \subset B(z, r)$, and thus (8) for $B(w, r / 2)$ yields (8) for $B(z, r)$, but now with a constant depending on $C$ and $\lambda$. We conclude, by Lemma 2, that (8) for all $w \in \partial \Omega$ implies the uniform $p$-fatness of $\Omega^{c}$.

Then let $w \in \partial \Omega$ and $r>0$. To prove that (8) holds, first assume that

$$
\left|B(w, r) \cap \Omega^{c}\right| \geq \frac{1}{4}|B(w, r)| .
$$

Let $\left\{B_{i}\right\}_{i=1}^{\infty}, B_{i}=B\left(z_{i}, r_{i}\right)$ for $z_{i} \in \Omega^{c}$ and $0<r_{i} \leq r$, be a covering of $B(w, r) \cap \Omega^{c}$. Then we have that

$$
\frac{1}{4} \leq \sum_{i}\left(\frac{r_{i}}{r}\right)^{n} \leq \sum_{i}\left(\frac{r_{i}}{r}\right)^{\lambda}
$$

and thus, by the definition of the $\lambda$-Hausdorff content, we see that (8) holds with constant $1 / 4$ under assumption (9).

We may hence assume that $|B(w, r) \cap \Omega| \geq \frac{3}{4}|B(w, r)|$. Then let $\left\{B_{i}\right\}_{i=1}^{\infty}$, $B_{i}=B\left(w_{i}, r_{i}\right)$ for $w_{i} \in \partial \Omega$ and $0<r_{i} \leq r$, be a covering of $B(w, r) \cap \partial \Omega$. If

$$
\sum_{i}\left|B_{i}\right| \geq \frac{1}{4} 2^{-n}|B(w, r)|
$$


it follows as in (10) that $\sum_{i} r_{i}^{\lambda} \geq C(n) r^{\lambda}$.

If (11) does not hold, i.e. we have that

$$
\sum_{i}\left|B_{i}\right|<\frac{1}{4} 2^{-n}|B(w, r)|
$$

we proceed as follows: Let $\hat{r}=(3 / 4)^{1 / n} r$ and denote $\alpha(n)=1-(3 / 4)^{1 / n}$, so that $r-\hat{r}=\alpha(n) r$. If there exists $x \in B(w, \hat{r}) \cap \Omega$ such that $d_{\Omega}(x) \geq \frac{1}{2} \alpha(n) r$, then, by the continuity of the distance function, there also exists $x^{\prime} \in B(w, \hat{r}) \cap \Omega$ such that $d_{\Omega}\left(x^{\prime}\right)=\frac{1}{2} \alpha(n) r$. Thus $B\left(x^{\prime}, 2 d_{\Omega}\left(x^{\prime}\right)\right) \subset B(w, r)$, and we obtain, by condition (3), that

$$
\mathcal{H}_{\infty}^{\lambda}(B(w, r) \cap \partial \Omega) \geq \mathcal{H}_{\infty}^{\lambda}\left(B\left(x^{\prime}, 2 d_{\Omega}\left(x^{\prime}\right)\right) \cap \partial \Omega\right) \geq C_{1} d_{\Omega}\left(x^{\prime}\right)^{\lambda} \geq C r^{\lambda},
$$

where $C=C\left(C_{1}, n, \lambda\right)>0$, and so (8) holds. We may hence assume that

$$
d_{\Omega}(x)<\frac{1}{2} \alpha(n) r \text { for every } x \in B(w, \hat{r}) \cap \Omega,
$$

so that in particular $B\left(x, 2 d_{\Omega}(x)\right) \subset B(w, r)$ for every $x \in B(w, \hat{r}) \cap \Omega$.

Let us denote $A=(B(w, \hat{r}) \cap \Omega) \backslash \bigcup_{i} 2 B_{i}$. We then have, by (12) and the choice of $\hat{r}$, that

$$
\begin{aligned}
|A| & \geq|B(w, \hat{r}) \cap \Omega|-\sum_{i} 2^{n}\left|B_{i}\right| \\
& \geq|B(w, r) \cap \Omega|-|B(w, r) \backslash B(w, \hat{r})|-2^{n} \frac{1}{4} 2^{-n}|B(w, r)| \\
& \geq \frac{3}{4}|B(w, r)|-\frac{1}{4}|B(w, r)|-\frac{1}{4}|B(w, r)| \geq \frac{1}{4}|B(w, r)| .
\end{aligned}
$$

Since $A \subset \bigcup_{x \in A} B\left(x, 6 d_{\Omega}(x)\right)$, we obtain, by a standard covering lemma (cf. [10]), a countable set of points $x_{k} \in A$ such that the corresponding balls $6 \tilde{B}_{k}$, where $\tilde{B}_{k}=B\left(x_{k}, d_{\Omega}\left(x_{k}\right)\right)$, are pairwise disjoint and $A \subset \bigcup_{k} 30 \tilde{B}_{k}$. Hence

$$
\frac{1}{4}|B(w, r)| \leq|A| \leq \sum_{k}\left|30 \tilde{B}_{k}\right| \leq 30^{n} \sum_{k}\left|\tilde{B}_{k}\right| .
$$

Since the radius of $\tilde{B}_{k}$ is $d_{\Omega}\left(x_{k}\right)<r$ for all $k$, and $\lambda \leq n$, it now follows from (14), similar to (10), that

$$
C(n) r^{\lambda} \leq \sum_{k} d_{\Omega}\left(x_{k}\right)^{\lambda} .
$$

When $i \in \mathbb{N}$, we let $\#_{i}$ denote the number of the balls $2 \tilde{B}_{k}$ such that $2 \tilde{B}_{k} \cap B_{i} \neq \emptyset$. But if $2 \tilde{B}_{k} \cap B_{i} \neq \emptyset$, then $d_{\Omega}\left(x_{k}\right)>\frac{1}{2} r_{i}$ (since $x_{k} \notin 2 B_{j}$ ), and thus $B_{i} \subset 6 \tilde{B}_{k}$. Since the balls $6 \tilde{B}_{k}$ are pairwise disjoint, it follows that $\#_{i} \leq 1$ for all $i \in \mathbb{N}$. Also, we have by (13) that $2 \tilde{B}_{k} \subset B(w, r)$, and so

$$
\mathcal{H}_{\infty}^{\lambda}\left(2 \tilde{B}_{k} \cap \partial \Omega\right) \leq \sum_{B_{i} \cap 2 \tilde{B}_{k} \neq \emptyset} r_{i}^{\lambda}
$$

for each $k$. Combining (15), (3), (16), and the fact that $\#_{i} \leq 1$, we finally obtain

$$
\begin{aligned}
r^{\lambda} & \leq C \sum_{k} d_{\Omega}\left(x_{k}\right)^{\lambda} \leq C \sum_{k} \mathcal{H}_{\infty}^{\lambda}\left(2 \tilde{B}_{k} \cap \partial \Omega\right) \\
& \leq C \sum_{k} \sum_{B_{i} \cap 2 \tilde{B}_{k} \neq \emptyset} r_{i}^{\lambda} \leq C \sum_{i} \#_{i} r_{i}^{\lambda} \leq C \sum_{i} r_{i}^{\lambda},
\end{aligned}
$$

where $C=C\left(C_{1}, n\right)>0$. Hence, by taking the infimum of the sums $\sum_{i} r_{i}^{\lambda}$ over all the coverings $\left\{B_{i}\right\}_{i}$ of $B(w, r) \cap \partial \Omega$, we see that equation (8) holds in this case as well. This also finishes the proof of Theorem 1 . 
Remark. From the proof of the part $(c) \Longrightarrow(a)$ of the theorem we obtain, with some minor modifications, the following result: Assume that a domain $\Omega \subset \mathbb{R}^{n}$ satisfies the inner boundary density condition (3) with exponent $\lambda$ and with a constant $C_{1}>0$, and let $0<\varepsilon<1$. Then, for each ball $B(w, r)$, where $w \in \partial \Omega$ and $r>0$, we have

$$
\left|B(w, r) \cap \Omega^{c}\right| \geq \varepsilon|B(w, r)| \text { or } \mathcal{H}_{\infty}^{\lambda}(B(w, r) \cap \partial \Omega) \geq C r^{\lambda},
$$

where $C=C\left(C_{1}, n, \lambda, \varepsilon\right)>0$. In particular, if there exists a constant $C_{2}>0$ such that $|B(w, r) \cap \Omega| \geq C_{2}|B(w, r)|$ for all $w \in \partial \Omega$ and $0<r<\operatorname{diam}(\Omega)$, we conclude that $\partial \Omega$ is $\lambda$-thick, with a constant $C=C\left(C_{1}, C_{2}, n, \lambda\right)>0$.

\section{An example}

We give a brief example in which we show that the $\lambda$-thickness of the boundary of $\Omega \subset \mathbb{R}^{n}$, for some $\lambda>n-p$, is not necessary for $\Omega$ to admit the pointwise $p$-Hardy inequality, or equivalently, for $\Omega$ to satisfy the inner boundary density condition (3) with the exponent $\lambda$.

Let $n, k \in \mathbb{N}$ be such that $n \geq 3$ and $1 \leq k \leq n-2$. Also let $\tau>1$. We consider the following domain $\Omega_{k} \subset \mathbb{R}^{n}$ :

$$
\Omega_{k}=\left\{\left(x_{1}, \ldots, x_{n}\right) \in \mathbb{R}^{n}: 0<x_{1}, \ldots, x_{k}<1, \sum_{i=k+1}^{n} x_{i}{ }^{n-k}<x_{1}^{\tau(n-k)}\right\} .
$$

Let $0<r<1$ and denote $B_{r}=B(0, r), E_{k, r}=\partial \Omega_{k} \cap B_{r}$. Then $E_{k, r}$ can be covered by approximately $r^{(1-\tau) k}$ balls of radius $r^{\tau}$. Now, if $\lambda>k$, we have that

$$
r^{-\lambda} \mathcal{H}_{\infty}^{\lambda}\left(E_{k, r}\right) \leq C r^{-\lambda} r^{(1-\tau) k} r^{\tau \lambda} \leq C r^{(\tau-1)(\lambda-k)} \longrightarrow 0
$$

as $r \rightarrow 0$, since $(\tau-1)(\lambda-k)>0$. This means that $\partial \Omega_{k}$ is not $\lambda$-thick for any $\lambda>k$. Nevertheless, it is obvious that the inner boundary density condition (3), with $\lambda=n-1$, holds for all $x \in \Omega_{k}$, and so $\Omega_{k}$ admits the pointwise $p$-Hardy inequality for all $p>1$, especially for $p=n-k$.

\section{ACKNOWLEDGMENTS}

The author wishes to express his gratitude to Professor Pekka Koskela for helpful discussions and valuable suggestions concerning the contents of this paper, and for reading the manuscript.

\section{REFERENCES}

[1] A. Ancona, 'On strong barriers and an inequality of Hardy for domains in $\mathbb{R}^{n}$, J. London Math. Soc. (2) 34 (1986), no. 2, 274-290. MR856511(87k:31004)

[2] P. HajŁasz, 'Pointwise Hardy inequalities', Proc. Amer. Math. Soc. 127 (1999), no. 2, 417423. MR 1458875 (99c:46028)

[3] G. H. Hardy, J. E. Littlewood and G. Pólya, 'Inequalities' (Second edition), Cambridge, at the University Press, 1952. MR0046395 (13:727e)

[4] J. Heinonen, T. Kilpeläinen and O. Martio, 'Nonlinear potential theory of degenerate elliptic equations', Oxford University Press, 1993. MR1207810 (94e:31003)

[5] J. Heinonen and P. Koskela, 'Quasiconformal maps in metric spaces with controlled geometry', Acta Math. 181 (1998), no. 1, 1-61. MR1654771(99j:30025)

[6] J. Kinnunen and O. Martio, 'Hardy's inequalities for Sobolev functions', Math. Res. Lett. 4 (1997), no. 4, 489-500. MR1470421 (98k:46052)

[7] P. Koskela and X. Zhong, 'Hardy's inequality and the boundary size', Proc. Amer. Math. Soc. 131 (2003), no. 4, 1151-1158. MR1948106 (2004e:26021) 
[8] J. L. Lewis, 'Uniformly fat sets', Trans. Amer. Math. Soc. 308 (1988), no. 1, 177-196. MR946438 (89e:31012)

[9] J. NEČAS, 'Sur une méthode pour résoudre les équations aux dérivées partielles du type elliptique, voisine de la variationnelle', Ann. Scuola Norm. Sup. Pisa (3) 16 (1962) 305-326. MR0163054 (29:357)

[10] E. M. Stein, 'Singular integrals and differentiability properties of functions', Princeton Mathematical Series, No. 30, Princeton University Press, Princeton, N.J., 1970. MR0290095 $(44: 7280)$

[11] A. Wannebo, 'Hardy inequalities', Proc. Amer. Math. Soc. 109 (1990), 85-95. MR 1010807 (90h:26025)

Department of Mathematics and Statistics, P.O. Box 35 (MaD), Fin-40014 University OF JYVÄSKYlä, Finland

E-mail address: juhaleh@maths.jyu.fi 